\title{
Crescimento e esporulação de isolados de Verticillium lecanii sob diferentes condições nutricionais ${ }^{(1)}$
}

\author{
Claudio Camargo Barbosa(2), Antonio Carlos Monteiro ${ }^{(2)}$, Antônia do Carmo Barcelos Correia(3) \\ e Gener Tadeu Pereira(4)
}

\begin{abstract}
Resumo - O objetivo deste trabalho foi determinar as melhores condições de crescimento e esporulação de dois isolados de Verticillium lecanii (Zimm.) Viégas, cultivados em vários meios de cultura, fontes de C, fontes orgânicas e inorgânicas de $\mathrm{N}$ e relações C:N. Após 20 dias de cultivo, BDA e Meio Completo mostraram ser os melhores meios de cultura, e a lactose e o amido foram as fontes de $\mathrm{C}$ que mais favoreceram o crescimento dos isolados JAB 02 e JAB 45, respectivamente; baixa esporulação foi apresentada por JAB 02 , enquanto JAB 45 obteve boa esporulação em glicose e maltose. JAB 02 desenvolveu melhor quando a fonte orgânica de $\mathrm{N}$ era casitona; JAB 45 obteve bom desenvolvimento em presença de casitona e peptona, mas houve pouca esporulação em presença de triptona e caseína hidrolisada. As fontes inorgânicas de $\mathrm{N}$ que favoreceram o melhor desenvolvimento dos isolados foram $\left(\mathrm{NH}_{4}\right)_{2} \mathrm{HPO}_{4}$ e $\mathrm{NaNO}_{3}$, porém, JAB 02 apresentou baixa esporulação. Entre as relações C:N analisadas, JAB 02 obteve melhor crescimento na relação 60:1 e JAB 45 nas relações 5:1, 10:1 e 20:1; baixa esporulação foi obtida por JAB 02 nas relações testadas, mas não se verificou efeito da relação $\mathrm{C}: \mathrm{N}$ do meio sobre a esporulação dos isolados.
\end{abstract}

Termos para indexação: fungos, meio de cultura, requisitos nutricionais, controle de pragas.

\section{Growth and sporulation of Verticillium lecanii isolates under different nutritional conditions}

Abstract - The objective of this work was to determine the best growth and sporulation conditions of two Verticillium lecanii (Zimm.) Viégas, isolates cultivated in different culture medium, C sources, organic and inorganic $\mathrm{N}$ sources and $\mathrm{C}: \mathrm{N}$ ratio. Twenty days after inoculation, PDA and Complete Medium provided the best development, and lactose and starch were the best $\mathrm{C}$ sources to promote the growth of JAB 02 and JAB 45 isolates, respectively; JAB 02 showed low sporulation while JAB 45 sporulated well on glucose and maltose. JAB 02 isolate developed well when the organic $\mathrm{N}$ source was casitone; JAB 45 obtained good development when the tested sources were casitone and peptone, although a low sporulation occurred in presence of tryptone and hydrolysed casein. The inorganic $\mathrm{N}$ sources that favoured the best development of isolates were $\left(\mathrm{NH}_{4}\right)_{2} \mathrm{HPO}_{4}$ and $\mathrm{NaNO}_{3}$, although JAB 02 isolate showed low sporulation. In the $\mathrm{C}: \mathrm{N}$ ratio analysed, JAB 02 isolate developed better on the $60: 1$ ratio, and the JAB 45 isolate on the 5:1, 10:1 and 20:1 ratio. The sporulation of JAB 02 isolate was low on all the ratios tested, but there was no effect of the $\mathrm{C}: \mathrm{N}$ ratio on the sporulation of both isolates.

Index terms: fungi, culture medium, nutritional requirements, pest control.

(1) Aceito para publicação em 24 de julho de 2001 . Extraído da dissertação de mestrado, apresentada pelo primeiro autor à Universidade Estadual Paulista (Unesp), Faculdade de Ciências Agrárias e Veterinárias (FCAV), Jaboticabal, SP.

(2) Unesp, FCAV, Dep. de Produção Vegetal, Via de Acesso Prof. Paulo Donato Castellane, s/n ${ }^{\circ}$, CEP 14884-900 Jaboticabal, SP. E-mail: ccbcamargo@uol.com.br, montecar@fcav.unesp.br

(3) Unesp, FCAV, Dep. de Fitossanidade. E-mail: antoniac@fcav.unesp.br

(4) Unesp, FCAV, Dep. de Ciências Exatas. E-mail: genertp@fcav.unesp.br

\section{Introdução}

A tendência do manejo de pragas está voltada para a preservação do meio ambiente, valendo-se de técnicas como o uso de bioinseticidas. Assim, o controle biológico com fungos entomopatogênicos é uma das alternativas mais importantes, com amplo potencial de utilização, sem deterioração dos recursos naturais (Padin et al., 1995).

Devido ao intenso efeito parasitário que produz sobre várias espécies de hospedeiros (Ignoffo et al., 
1976; Ferron, 1981; Alves, 1998) Verticillium lecanii (Zimm.) Viégas é considerado um dos agentes fúngicos com maiores possibilidades no controle microbiano de insetos (Lecuona \& Riba, 1991).

Esse fungo, além de ser um importante patógeno de homópteros como afídeos, coccídeos e aleirodídeos (Khalil \& Táborský, 1982; Grajek, 1994), causa doença em algumas espécies de ácaros, e pode ser hiperparasito de fungos fitopatogênicos, como várias ferrugens (Castaldi \& Nicoli, 1993). Há relatos de sucesso de seu uso, tanto em casa de vegetação como no campo (Khalil et al., 1985). Porém, a produção de esporos do fungo pode variar consideravelmente, o que causa problemas na obtenção de conídios para o controle biológico (Verhaar \& Hijwegen, 1993).

Um fator importante na produção massal do fungo é a seleção de um meio-padrão para o cultivo de uma espécie ou de um isolado em particular, e no conhecimento das condições adequadas de cultivo que possibilitem a esta espécie, linhagem ou isolado, obter bom crescimento com alta esporulação (Khalil et al., 1985).

O cultivo de $V$. lecanii em meios sólidos tem sido utilizado com sucesso (Grajek, 1994). Um dos fatores mais importantes para o estabelecimento de um bom meio nutritivo sólido é a utilização de fontes adequadas de $\mathrm{C}$ e de $\mathrm{N}$, bem como uma relação otimizada entre estes dois nutrientes.

Muitos fungos utilizam glicose como fonte de C, porém vários outros açúcares podem ser melhor aproveitados por um isolado em particular (Carlile \& Watkinson, 1994). Além disso, uma fonte de N prontamente assimilável é indispensável para o desenvolvimento fúngico (Garraway \& Evans, 1984), e um equilíbrio apropriado entre estes nutrientes no meio é um fator importante para o crescimento e esporulação de fungos (Lilly \& Barnett, 1951).

O objetivo deste trabalho foi avaliar os efeitos de diversos meios de cultura, de diferentes fontes de $\mathrm{C}$ e $\mathrm{N}$ e da relação $\mathrm{C}: \mathrm{N}$ no crescimento radial e na esporulação de dois isolados de $V$. lecanii.

\section{Material e Métodos}

Foram utilizados dois isolados de Verticillium lecanii: 0 JAB 02, obtido da cochonilha-verde Coccus viridis Green
(Hemiptera: Coccidae), em citros, no Município de Ubirajara, SP, cedido pelo Laboratório de Entomopatógenos do Departamento de Defesa Fitossanitária da FCAV/ Unesp, Campus de Jaboticabal, e o JAB 45, também obtido de C. viridis, em citros, porém no Município de São Carlos, SP. Os isolados foram cultivados em tubos de ensaio contendo meio de batata-dextrose-agar (BDA), por 10 dias, a $27 \pm 0,5^{\circ} \mathrm{C}$, e fotófase de 14 horas, e as culturas foram estocadas em refrigerador a $4^{\circ} \mathrm{C}$.

Foram preparadas placas de Petri contendo o meio BDA, onde foram inoculadas alíquotas do fungo retiradas da cultura-estoque, e incubadas a $27 \pm 0,5^{\circ} \mathrm{C}$, com fotófase de 14 horas, por 20 dias. Culturas assim rejuvenescidas serviram de inóculo para os ensaios.

O cultivo foi realizado em placas de Petri de 12 × $80 \mathrm{~mm}$ contendo $15 \mathrm{~mL}$ de meio de cultura. A inoculação foi efetuada com agulha de níquel-cromo por meio de simples picada, transferindo-se esporos e fragmentos de micélio para o ponto central das placas. Após inoculação, o fungo foi incubado em estufa a $27 \pm 0,5^{\circ} \mathrm{C}$, por 20 dias, no escuro.

A avaliação do crescimento radial da colônia foi realizada com régua milimétrica, medindo-se, a cada dois dias, por um período de 20 dias, dois diâmetros previamente marcados na face externa do fundo da placa de Petri. A avaliação da produção de esporos foi realizada no $20^{\circ}$ dia de incubação. Para tanto, escolheram-se, ao acaso, três placas de cada tratamento, retirando-se de cada uma, com auxílio de um furador de rolha metálico, três discos de $8 \mathrm{~mm}$ de diâmetro, sendo um da região central, outro da região mediana e outro da região periférica das colônias. Os discos foram transferidos isoladamente para tubos de ensaio contendo $10 \mathrm{~mL}$ de uma mistura (1:1) de solução salina $(\mathrm{NaCl}$ a $0,89 \% \mathrm{p} / \mathrm{v})$ e solução de Tween 80 $(0,1 \% \mathrm{v} / \mathrm{v})$. Por meio de vigorosa agitação em agitador elétrico de tubos, extraíram-se os conídios, e procedeu-se à contagem em Câmara de Neubauer.

Cinco ensaios foram organizados, para avaliar o desempenho do fungo em relação a diferentes condições de cultivo. No primeiro, avaliou-se o crescimento e esporulação dos isolados em diferentes meios de cultura. Utilizou-se Meio de Sabouraud (MS) (Aaronson, 1970), Meio Completo (MC) (Pontecorvo et al., 1953, modificado por Azevedo \& Costa, 1973), BDA, Meio de Czapeck-Dox (MCD) (Aaronson, 1970) e Meio Mínimo (MM) (Pontecorvo et al., 1953).

No segundo ensaio, estudou-se a utilização de diferentes açúcares (glicose, sacarose, maltose, lactose e amido), adicionadas na quantidade de $10 \mathrm{~g} / \mathrm{L}$, como fontes de carbono.

No terceiro e no quarto, verificou-se o desempenho dos isolados cultivados em meio contendo diferentes fon- 
tes orgânicas ou inorgânicas de nitrogênio. Como fonte orgânica, utilizaram-se peptona, triptona, casitona, caseína hidrolizada e extrato de carne, adicionadas na quantidade de $10 \mathrm{~g} / \mathrm{L}$. Como fonte inorgânica, utilizaram-se $\mathrm{NaNO}_{3}$, $\mathrm{NH}_{4} \mathrm{NO}_{3},\left(\mathrm{NH}_{4}\right)_{2} \mathrm{SO}_{4},\left(\mathrm{NH}_{4}\right)_{2} \mathrm{HPO}_{4}$ e $\mathrm{NH}_{4} \mathrm{Cl}$, adicionados na quantidade de $6 \mathrm{~g} / \mathrm{L}$. Nestes ensaios substituíu-se a fonte original dos meios de cultivo pela fonte orgânica ou inorgânica de $\mathrm{N}$ em análise.

No quinto, verificou-se o desempenho dos isolados cultivados sob o efeito de diferentes relações C:N (5:1, 10:1, 20:1, 40:1 e 60:1) do meio de cultura.

Para a condução dos quatro últimos ensaios, o isolado JAB 02 foi cultivado no Meio de Czapeck-Dox; usou-se maltose como fonte de $\mathrm{C}$ nos três últimos, e para o estabelecimento das relações $\mathrm{C}: \mathrm{N}$, utilizou-se $\mathrm{NaNO}_{3}$ como fonte de N. Da mesma forma, o isolado JAB 45 foi cultivado no Meio Mínimo, usando glicose como fonte de $\mathrm{C}, \mathrm{e} \mathrm{NaNO}_{3}$ como fonte de $\mathrm{N}$ para a relação $\mathrm{C}: \mathrm{N}$.

Os ensaios foram conduzidos segundo o delineamento inteiramente casualizado, com cinco tratamentos. Para avaliação do crescimento, foram feitas seis repetições (seis placas por tratamento) e os dados foram analisados por meio de regressão linear, seguida de Teste de Paralelismo (teste $\mathrm{t}$ ) e coincidência (teste F) entre as retas (Dixon \& Massey Junior, 1969). Para a produção de esporos, foram efetuadas três repetições (três placas por tratamento) Os dados foram submetidos à análise de variância pelo teste $\mathrm{F}$, a $1 \%$ de probabilidade, e as médias, comparadas pelo teste de Tukey, a $5 \%$ de probabilidade.

\section{Resultados e Discussão}

O melhor meio de cultura para o crescimento do isolado JAB 02 foi o de Sabouraud, que diferiu estatisticamente dos demais meios, seguido dos Meios de BDA e Completo, que não diferiram entre si $\left(\mathrm{F}=0,62^{\mathrm{ns}} ; \mathrm{t}=-0,04^{\mathrm{ns}}\right)$. Para o crescimento do isolado JAB 45, os melhores Meios foram Sabouraud e Completo, que não diferiram significativamente entre si $\left(F=2,29^{\text {ns }} ; t=0,78^{\text {ns }}\right)$. Diferenças significativas foram observadas principalmente em relação ao desempenho dos isolados cultivados nos Meios salinos de Czapeck-Dox e Mínimo, onde se obtiveram os menores crescimentos (Figura 1). Resultados diferentes foram observados na produção de esporos (Tabela 1). O isolado JAB 02 apresentou melhor esporulação quando cultivado em BDA, ao passo que, em Meio de Sabouraud, a produção de esporos foi significativamente inferior. Em relação ao JAB 45, os Meios Completo, Mínimo e BDA se mostraram mais favoráveis à produção de esporos e, como no
JAB 02, a esporulação foi significativamente inferior no Meio de Sabouraud. Tanto o menor crescimento como a melhor esporulação ocorreram no Meio Mínimo (Figura 1 e Tabela 1).
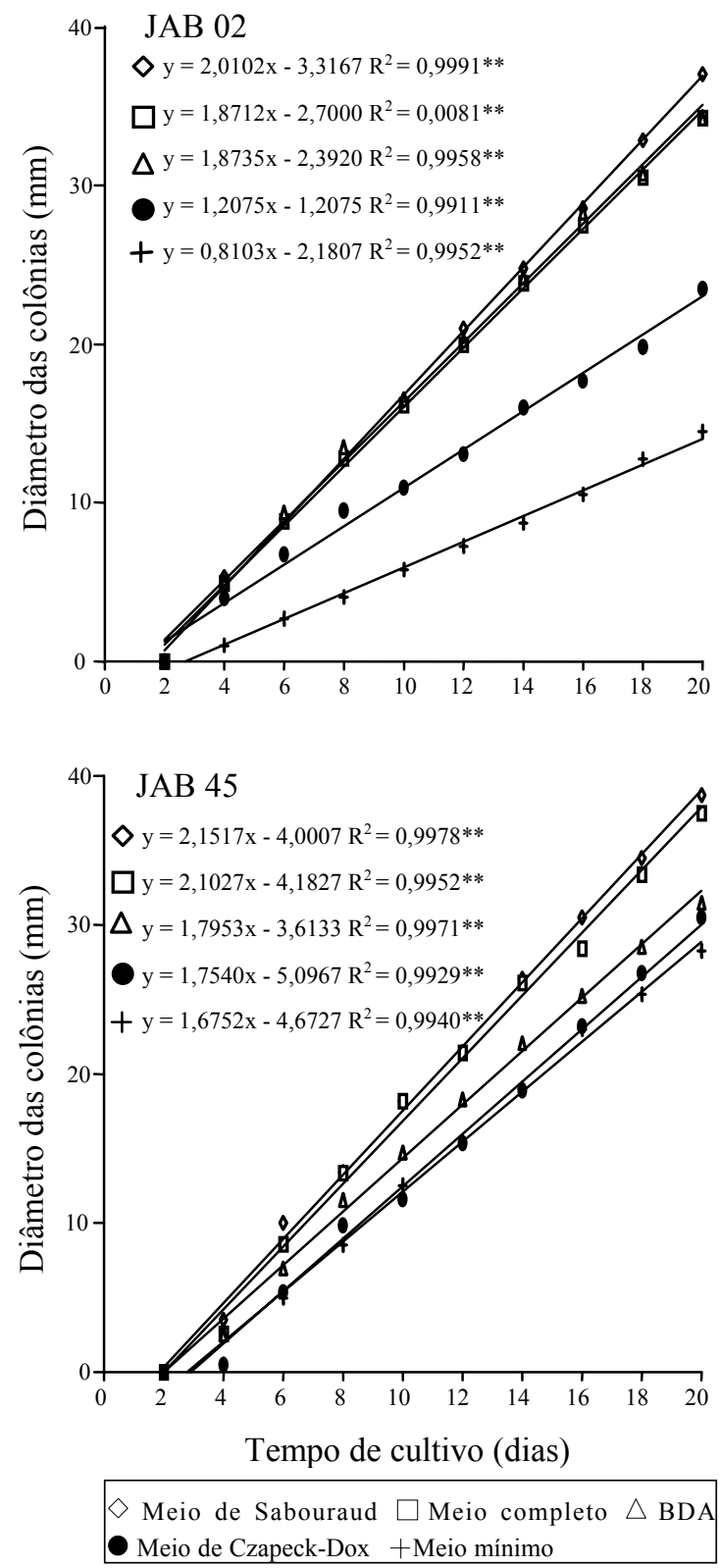

Figura 1. Crescimento radial de Verticillium lecanii cultivado em diversos meios de cultura. $* *$ Significativo a $1 \%$ de probabilidade pelo teste $\mathrm{F}$. 
Os resultados obtidos em Meio Completo se devem, provavelmente, à sua composição: peptona, extrato de levedura e caseína hidrolisada, que contêm vários macro e micronutrientes necessários ao desenvolvimento do fungo, além de vitaminas que atuam como fatores auxiliares. No Meio de Sabouraud, a ausência de algum fator necessário à formação dos conídios foi, provavelmente, responsável pela baixa esporulação verificada. Grajek (1994), testando a esporogenicidade de $V$. lecanii em diferentes meios de cultura, obteve boa produção de esporos em Meio de Sabouraud suplementado com farelo de trigo, polpa de beterraba ou com a mistura de ambos. Neste caso, as suplementações podem ter suprido o fungo com o fator que estava faltando. Por outro lado, a limitada disponibilidade de nutrientes presentes no Meio Mínimo pode ter induzido a esporulação de JAB 45; entretanto, este fato não ocorreu com JAB 02 , e provavelmente é conseqüência da variabilidade genética entre os isolados.

Os resultados de crescimento e esporulação de fungos entomopatogênicos encontrados na literatura são bastante diversificados, o que pode ser atribuído à variabilidade existente entre os isolados, ou até, aos diferentes métodos de avaliação utilizados.

Khalil \& Táborský (1982) avaliaram o crescimento e esporulação de $V$. lecanii em vários meios de cultura. Após 20 dias de cultivo, os maiores crescimentos radiais das colônias foram obtidos nos Meios Malte-Lactose-Peptona (72,33 mm), Czapeck-Dox (71,00 mm) e Sabouraud (63,66 mm). A produção de esporos foi avaliada 96 horas após a inoculação, e as maiores produções foram observadas nos Meios Malte-Lactose-Peptona $\left(26,8 \times 10^{8}\right.$ con. $\left./ \mathrm{mL}\right)$ e

Tabela 1. Esporulação dos isolados JAB 02 e JAB 45 de Verticillium lecanii $\left(\mathrm{n}^{0}\right.$ de conídios x $\left.10^{4} / \mathrm{mL}\right)$ no vigésimo dia de cultivo em diversos meios de cultura ${ }^{(1)}$.

\begin{tabular}{lcc}
\hline Meio de cultura & JAB 02 & JAB 45 \\
\hline Sabouraud & $4,55 \mathrm{c}$ & $20,28 \mathrm{c}$ \\
Completo & $19,44 \mathrm{~b}$ & $106,67 \mathrm{a}$ \\
BDA & $51,00 \mathrm{a}$ & $79,72 \mathrm{ab}$ \\
Czapeck-Dox & $2,00 \mathrm{c}$ & $48,34 \mathrm{bc}$ \\
Mínimo & $1,00 \mathrm{c}$ & $105,28 \mathrm{a}$ \\
\hline CV $(\%)$ & 23,68 & 28,20 \\
\hline
\end{tabular}

${ }^{(1)}$ Médias seguidas de mesma letra, na coluna, não diferem entre si pelo teste de Tukey, a 5\% de probabilidade.
Sabouraud (23,7 x $\left.10^{8} \mathrm{con} . / \mathrm{mL}\right)$. Balazy (1963), citado por Khalil \& Táborský (1982), obteve bom crescimento e esporulação de $V$. lecanii nos Meios Czapeck-Dox, Malte e BDA.

Hsiao et al. (1992) constataram que Meios de composição definida acrescidos com Dopamina, Dopamina- $\mathrm{HCl}$ ou Norepinefrina-HCl propiciaram melhor desenvolvimento de um isolado de $V$. lecanii do que o meio de Extrato de Levedura-Peptona-Dextrose.

JAB 02 e JAB 45 apresentaram maiores crescimentos em meios onde as fontes de $\mathrm{C}$ eram lactose $\mathrm{e}$ amido, respectivamente, embora para o último isolado o teste de paralelismo não tenha apresentado diferença significativa entre amido e as demais fontes de C, como glicose (Figura 2). A produção de esporos por JAB 02 foi muito baixa em todas as fontes de $\mathrm{C}$ testadas, e não se observou diferença estatística entre elas. O isolado JAB 45 apresentou melhor esporulação em presença de glicose, maltose e sacarose; não se observou diferença significativa entre estas três, mas sim entre as cinco fontes avaliadas (Tabela 2).

Pelo fato de a glicose ser uma molécula prontamente utilizável, muitos autores a consideram como a melhor fonte de $\mathrm{C}$ para o crescimento e esporulação de várias espécies de fungos entomopatogênicos. No presente ensaio este resultado foi confirmado apenas em relação ao isolado JAB 45. Apesar de a lactose ser caracterizada como fonte de $\mathrm{C}$ pobre para suportar o crescimento fúngico (Cochrane, 1958), foi o carboidrato que forneceu a melhor condição para o crescimento de JAB 02 , em virtude, provavelmente, da adaptação metabólica do isolado a ela.

Coetzee \& Eicker (1991) compararam 15 fontes de $\mathrm{C}$ para determinar o efeito de fatores nutricionais e ambientais no crescimento e esporulação de Verticillium fungicola. As melhores fontes foram glicose, manitol e sacarose.

Monteiro (1988) testou quatro fontes de C (glicose, sacarose, amido e lactose) para o crescimento e produção de esporos por isolados de Metarhizium anisopliae, Beauveria bassiana e Paecilomyces marquandii. Glicose e amido foram os melhores açúcares para o crescimento de $M$. anisopliae e B. bassiana, enquanto glicose ou sacarose foram as melhores fontes de $\mathrm{C}$ para o cres- 
cimento e esporulação de $P$. marquandii, após 10 dias de cultivo. Para M. anisopliae e B. bassiana a mais expressiva produção de esporos foi obtida ape-
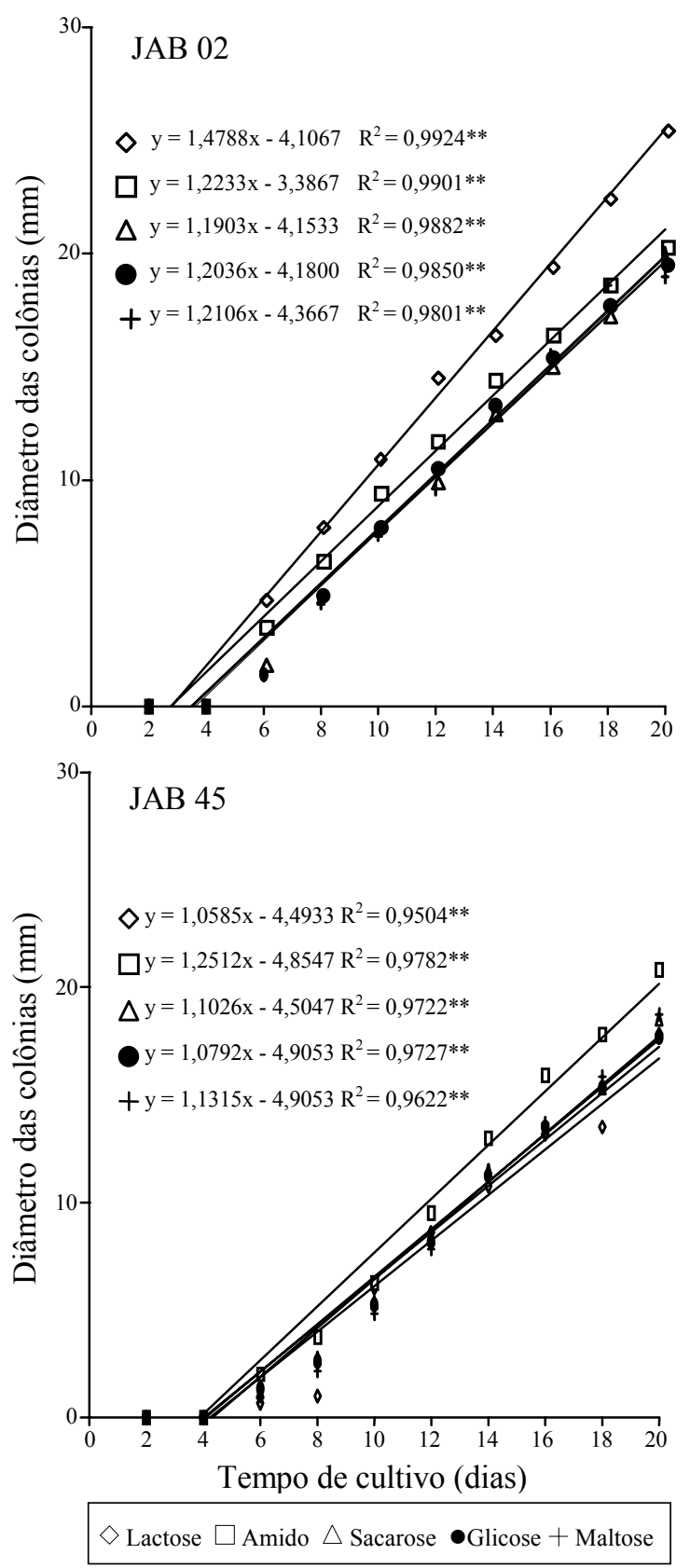

Figura 2. Crescimento radial de Verticillium lecanii cultivado em diversas fontes de carbono. ${ }^{* *}$ Significativo a $1 \%$ de probabilidade pelo teste $\mathrm{F}$. nas quando amido era a fonte única de carbono.

Não se verificou diferença estatística entre as fontes orgânicas de $\mathrm{N}$ para o crescimento do isolado JAB 02 (Figura 3) e para sua esporulação, as melhores fontes foram casitona, peptona e extrato de carne (Tabela 2). As melhores fontes para o crescimento do isolado JAB 45 foram casitona, peptona e triptona, que não diferiram entre si pelo teste de paralelismo (Figura 3). Em relação à produção de esporos, as melhores fontes orgânicas de $\mathrm{N}$ foram extrato de carne, casitona e peptona (Tabela 2).

Tabela 2. Esporulação dos isolados JAB 02 e JAB 45 de Verticillium lecanii $\left(\mathrm{n}^{0}\right.$ de conídios $\left.\times 10^{4} / \mathrm{mL}\right)$ no vigésimo dia de cultivo em diversas fontes de carbono, fontes orgânicas e inorgânicas de nitrogênio e em meios de cultivo com diferentes relações $\mathrm{C}: \mathrm{N}^{(1)}$.

\begin{tabular}{|c|c|c|}
\hline Fator nutricional & JAB 02 & JAB 45 \\
\hline \multicolumn{3}{|l|}{ Fonte de carbono } \\
\hline Lactose & $0,67 \mathrm{a}$ & $4,00 \mathrm{c}$ \\
\hline Amido & $1,33 \mathrm{a}$ & $4,67 b c$ \\
\hline Sacarose & $0,33 \mathrm{a}$ & $8,33 \mathrm{abc}$ \\
\hline Glicose & $1,00 \mathrm{a}$ & $14,33 \mathrm{a}$ \\
\hline Maltose & $1,33 \mathrm{a}$ & $11,67 \mathrm{ab}$ \\
\hline CV $(\%)$ & 87,48 & 32,61 \\
\hline \multicolumn{3}{|c|}{ Fonte orgânica de nitrogênio } \\
\hline Casitona & $38,78 \mathrm{a}$ & $63,00 \mathrm{ab}$ \\
\hline Extrato de carne & $27,22 \mathrm{a}$ & $95,00 \mathrm{a}$ \\
\hline Peptona & $37,89 \mathrm{a}$ & $58,00 \mathrm{ab}$ \\
\hline Triptona & $1,55 \mathrm{~b}$ & $48,33 b$ \\
\hline Caseína hidrolisada & $0,00 \mathrm{~b}$ & $48,67 \mathrm{~b}$ \\
\hline $\mathrm{CV}(\%)$ & 40,01 & 22,33 \\
\hline \multicolumn{3}{|c|}{ Fonte inorgânica de nitrogênio } \\
\hline Nitrato de sódio & $0,00 \mathrm{a}$ & $56,67 \mathrm{~b}$ \\
\hline Fosfato de amônio & $0,00 \mathrm{a}$ & $132,33 \mathrm{a}$ \\
\hline Cloreto de amônio & $0,33 \mathrm{a}$ & $3,00 \mathrm{c}$ \\
\hline Sulfato de amônio & $0,00 \mathrm{a}$ & $4,33 \mathrm{c}$ \\
\hline Nitrato de amônio & $0,33 \mathrm{a}$ & $16,00 \mathrm{c}$ \\
\hline CV $(\%)$ & 273,86 & 28,49 \\
\hline \multicolumn{3}{|l|}{ Relação C:N } \\
\hline $5: 1$ & $4,67 \mathrm{a}$ & $57,11 \mathrm{a}$ \\
\hline $10: 1$ & $2,33 \mathrm{a}$ & $72,56 \mathrm{a}$ \\
\hline $20: 1$ & $1,67 \mathrm{a}$ & $74,78 \mathrm{a}$ \\
\hline $40: 1$ & $0,33 \mathrm{a}$ & $82,33 a$ \\
\hline $60: 1$ & $1,00 \mathrm{a}$ & $53,00 \mathrm{a}$ \\
\hline CV (\%) & 98,32 & 27,62 \\
\hline
\end{tabular}

(1)Médias seguidas de mesma letra, na coluna, não diferem entre si pelo teste de Tukey, a 5\% de probabilidade. 
Resultados diferentes destes foram obtidos com M. anisopliae e B. bassiana em meios contendo diversas fontes orgânicas de $\mathrm{N}$ (Barnes et al., 1975).
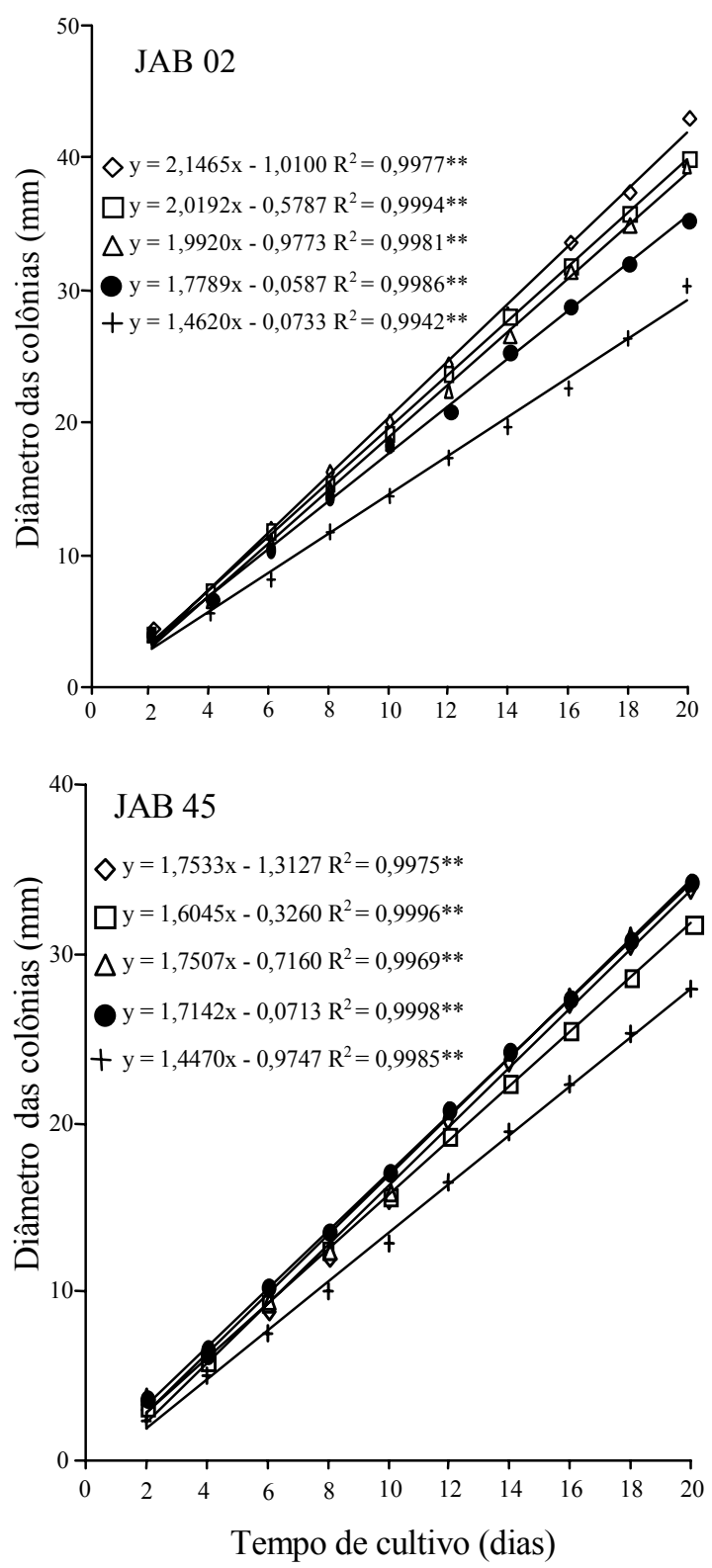

$\diamond$ Casitona $\square$ Extrato de carne $\triangle$ Peptona Triptona + Caseína hidrolisada

Figura 3. Crescimento radial de Verticillium lecanii cultivado em diversas fontes orgânicas de nitrogênio. **Significativo a $1 \%$ de probabilidade pelo teste $\mathrm{F}$.
Triptona e casitona mostraram-se as melhores fontes de $\mathrm{N}$ orgânico para o crescimento de M. anisopliae, enquanto soitona foi a melhor fonte para o crescimento de B. bassiana. Caseína hidrolisada (M. anisopliae) e peptona (M. anisopliae e $B$. bassiana) apresentaram os piores resultados no crescimento fúngico. $M$. anisopliae obteve regular esporulação em meios acrescidos de triptona e casitona, porém em meios acrescidos de caseína hidrolizada ou peptona, a esporulação mostrou-se muito inferior às demais. B. bassiana obteve boa esporulação em triptona e casitona, baixa esporulação em caseína hidrolisada, e muito baixa esporulação em peptona.

O maior crescimento radial de JAB 02 foi obtido quando se utilizou nitrato de sódio como fonte de $\mathrm{N}$ no meio, e JAB 45 apresentou melhores resultados em nitrato de sódio e fosfato de amônio di-básico, que não diferiram significativamente entre si pelos testes de paralelismo e coincidência de retas (Figura 4). As fontes inorgânicas de $\mathrm{N}$ propiciaram bom crescimento dos isolados. Por outro lado, muito baixa ou nenhuma produção de esporos foi obtida por JAB 02 , e o isolado JAB 45 apresentou boa esporulação apenas em fosfato de amônio di-básico (Tabela 2).

JAB 45 apresentou bom desempenho em presença de sais com o íon amônio, sendo que o melhor crescimento e esporulação foi obtido em presença de fosfato de amônio di-básico, sugerindo, assim, que a presença conjunta dos íons fosfato e amônio tenha favorecido seu desenvolvimento. O maior crescimento obtido pelo JAB 02 foi em nitrato de sódio, porém sua esporulação foi praticamente nula em presença dos vários sais nitrogenados.

$\mathrm{O}$ crescimento de JAB 02 foi melhor na relação 60:1; e pior, nas relações 5:1 e 10:1, enquanto JAB 45 obteve os maiores crescimentos nas relações 10:1, 5:1 e 20:1, que não diferiram estatisticamente entre si pelo teste de paralelismo (Figura 5). A produção de esporos do isolado JAB 02 foi baixa em todas as relações testadas, ao passo que, comparativamente a esta, a esporulação de JAB 45 foi melhor. Entretanto, não se verificou efeito da relação $\mathrm{C}$ : $\mathrm{N}$ do meio de cultura sobre a esporulação de ambos os isolados (Tabela 2). 
Meios de cultura com relação C:N $\leq 10: 1$ resultam na formação de micélio com elevada concentração de proteínas e altas relações $\mathrm{C}: \mathrm{N}$, como, por exem-
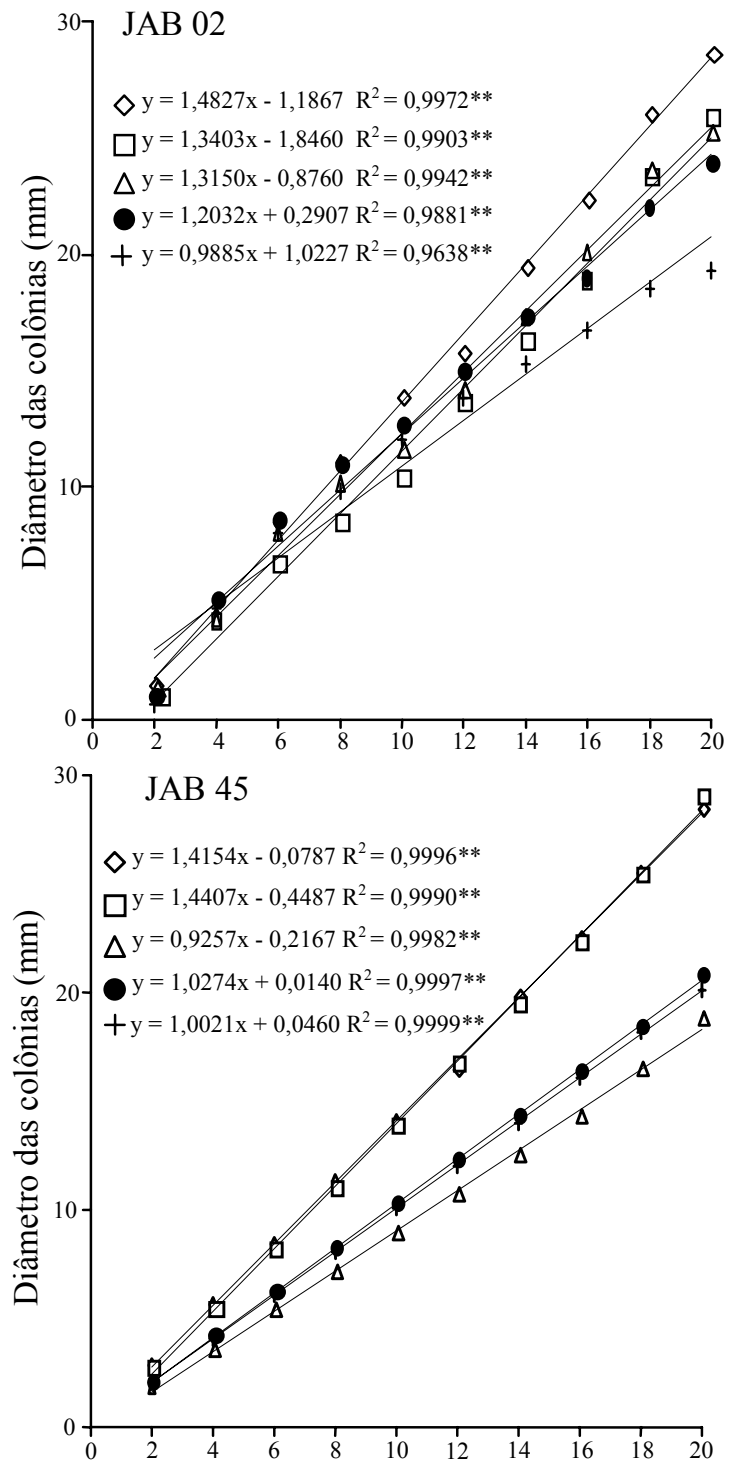

Tempo de cultivo (dias)

$\diamond \mathrm{NaNO}_{3} \quad \square\left(\mathrm{NH}_{4}\right)_{2} \mathrm{HPO}_{4} \triangle \mathrm{NH}_{4} \mathrm{Cl}$

- $\left(\mathrm{NH}_{4}\right)_{2} \mathrm{SO}_{4}+\mathrm{NH}_{4} \mathrm{NO}_{3}$

Figura 4. Crescimento radial de Verticillium lecanii cultivado em diversas fontes inorgânicas de nitrogênio. **Significativo a $1 \%$ de probabilidade pelo teste $\mathrm{F}$. plo, 50:1, e favorecem o acúmulo de álcool, metabólitos secundários, lipídeos e polissacarídeos extracelulares pelo fungo (Carlile \& Watkinson, 1994). Porém, os resultados deste trabalho mostraram que
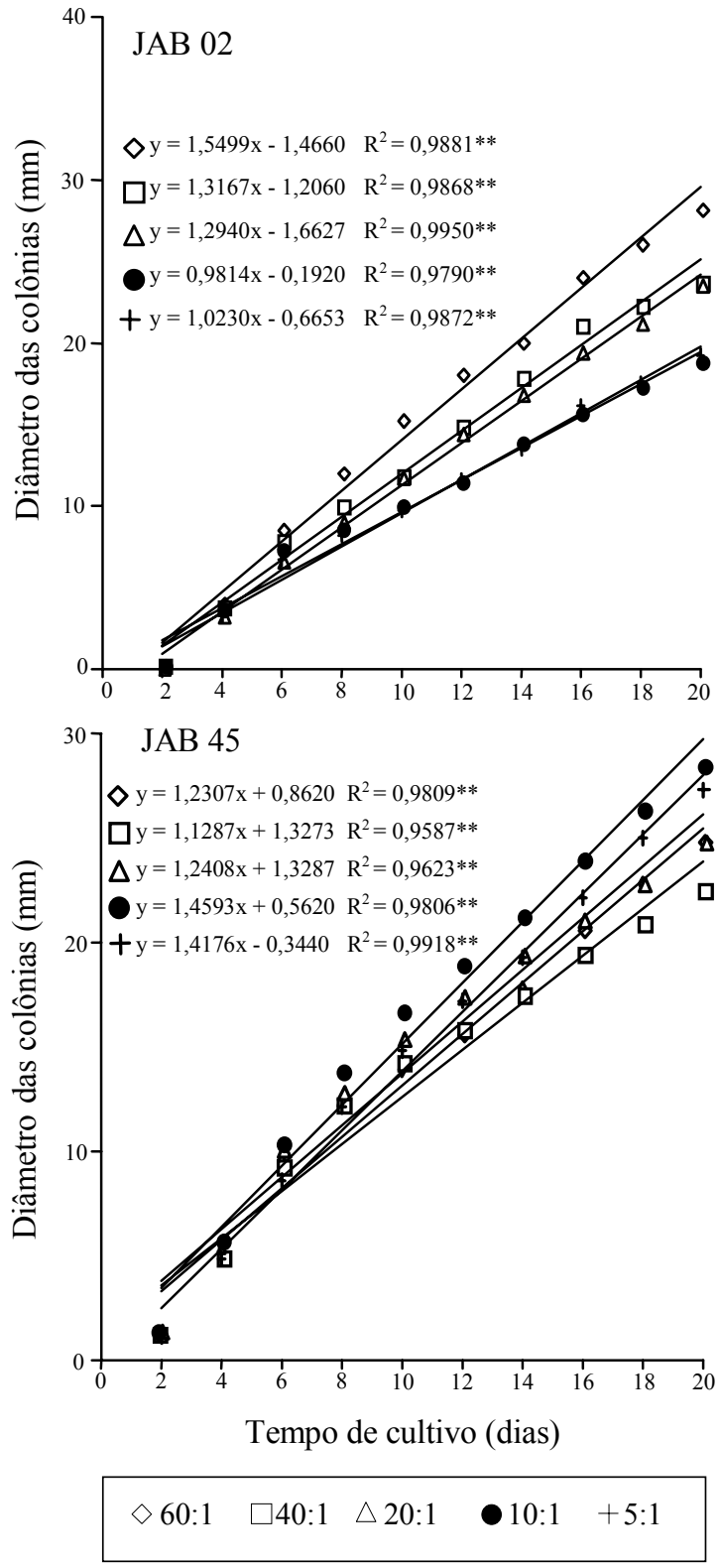

Figura 5. Crescimento radial de Verticillium lecanii cultivado em diversas relações $\mathrm{C}: \mathrm{N}$. ${ }^{* *}$ Significativo a $1 \%$ de probabilidade pelo teste $\mathrm{F}$. 
ambos os isolados se desenvolveram nestas condições, ou seja, JAB 02 apresentou os melhores resultados na relação 60:1 e JAB 45, nas relações 10:1 e 5:1.

\section{Conclusões}

1. Os isolados JAB 02 e JAB 45 apresentam crescimento radial e esporulação diferentes, quando cultivados em meios diversos.

2. As fontes de $\mathrm{C}$ têm efeito no desempenho dos dois isolados, determinando variações no crescimento e na esporulação.

3. O crescimento dos isolados é influenciado tanto pelas fontes orgânicas quanto inorgânicas de N, mas ocorrem amplas variações com relação à esporulação.

4. A relação C:N do meio de cultura influencia significativamente o crescimento, mas não a esporulação dos isolados.

\section{Agradecimentos}

À Coordenadoria de Aperfeiçoamento de Pessoal de Ensino Superior (Capes), pelo apoio financeiro, através da concessão da bolsa de mestrado.

\section{Referências}

AARONSON, S. Experimental microbial ecology. New York: Academic, 1970.236 p.

ALVES, S. B. Fungos entomopatogênicos. In: ALVES, S. B. (Ed.). Controle microbiano de insetos. 2. ed. Piracicaba: Esalq, 1998. p. 289-382.

AZEVEDO, J. L.; COSTA, S. O. P. (Ed.). Exercícios práticos de genética. São Paulo: Editora Nacional/Edusp, 1973. $288 \mathrm{p}$.

BARNES, G. L.; BOETHEL, D. L.; EIKENBARY, R. D.; CRISWELL, J. T.; GENTRY, C. R. Growth and sporulation of Metarhizium anisopliae and Beauveria bassiana on media containing various peptone sources. Journal of Invertebrate Pathology, San Diego, v. 25, p. 301-305, 1975.

CARLILE, M. J.; WATKINSON, S. C. The fungi. San Diego: Academic, 1994. 428 p.
CASTALDI, R.; NICOLI, G. Verticillium lecanii. Informatore Fitopatologico, Bologna, v. 43, n. 10, p. 2024, 1993.

COCHRANe, V. W. Physiology of fungi. New York: J. Wiley, 1958. $524 \mathrm{p}$.

COETZEE, J. C.; EICKER, A. The effect of nutritional and environmental factors on the growth and sporulation of a Southern African isolate of Verticillium fungicola. In: INTERNATIONAL CONGRESS ON THE SCIENCE AND CULTIVATION OF EDIBLE FUNGI, 13., 1991, Dublin. Proceedings... Dublin: Balkema, 1991. v. 2, p. 417-424.

DIXON, W. J.; MASSEY JUNIOR, F. J. Introduction to statistical analysis. 3. ed. Tokyo: McGraw-Hill Kogakusha, 1969. 638 p.

FERRON, P. Pest control by the fungi Beauveria bassiana. In: BURGES, H. D. (Ed.). Microbial control of pest and plant diseases. London: Academic, 1981. p. 465-482.

GARRAWAY, M. O.; EVANS, R. C. Fungal nutrition and physiology. New York: J. Wiley, 1984. 401 p.

GRAJEK, W. Sporogenesis of the entomopathogenic fungus Verticillium lecanii in solid-state cultures. Folia Microbiologica, London, v. 39, n. 1, p. 29-32, 1994.

HSIAO, W. F.; BIDOCHKA, M. J.; KHACHATOURIANS, G. G. Effects of diphenols on the growth of three entomopathogenic fungi. Canadian Journal of Microbiology, Ottawa, v. 38, p. 1000-1003, 1992.

IGNOFFO, C. M.; PUTTLER, B.; HOSTETTER, D. L.; DICKERSON, W. A. Susceptibility of the cabbage hooper, Trichoplusiani, and the velvetbean caterpillar, Anticarsia gemmatalis, to several isolates of the entomopathogenic fungus Nomuraea rileyi. Journal of Invertebrate Pathology, San Diego, v. 28, p. 259-262, 1976.

KHALIL, S. K.; SHAH, M. A.; NAEEM, M. Laboratory studies on the compatibility of the entomopathogenic fungus Verticillium lecanii with certain pesticides. Agriculture, Ecosystems and Environment, Amsterdam, v. 13 , p. $329-334,1985$.

KHALIL, S. K.; TÁBORSKÝ, V. The effect of different media on the growth and sporulation of the entomopathogenic fungus Verticillium lecanii (Zimm.) Viégas. Agricultura Tropica et Subtropica, Praga, v. 15, p. 251-268, 1982.

LECUONA, R. E.; RIBA, G. Primeras etapas del ciclo de desarrollo de hongos entomopatógenos. Pergamino: 
Instituto Nacional de Tecnología Agropecuaria, 1991.30 p. (Boletín de Divulgación Tecnológica, 87).

LILLY, V. G.; BARNETT, H. L. Physiology of the fungi. New York: McGraw-Hill Book, 1951. 464 p.

MONTEIRO, A. C. Aspectos fisioecológicos de isolados de fungos entomopatogênicos obtidos na região amazônica (Manaus). 1988. 233 f. Tese (Doutorado) Universidade Federal de São Carlos, São Carlos.

PADIN, S. B.; DAL BELLO, G. M.; VASICEK, A. L. Potencial bioinsecticida de hongos entomopatogenos de plagas en granos almacenados. Revista de la Facultad de Agronomía, Buenos Aires, v. 15, n. 1, p. 1-7, 1995.

PONTECORVO, G.; ROPER, J. A.; HEMMONS, L. M.; MacDONALD, K. D.; BUFTON, A. W. J. The genetics of Aspergillus nidulans. Advances in Genetics, San Diego, v. 5, p. 141-238, 1953.

VERHAAR, M. A.; HIJWEGEN, T. Efficient production of phialoconidia of Verticillium lecanii for biocontrol of cucumber powdery mildew, Sphaerotheca fuliginea.

Netherlands Journal of Plant Pathology, Dordrecht, v. 99, n. 2, p. 101-103, 1993. 